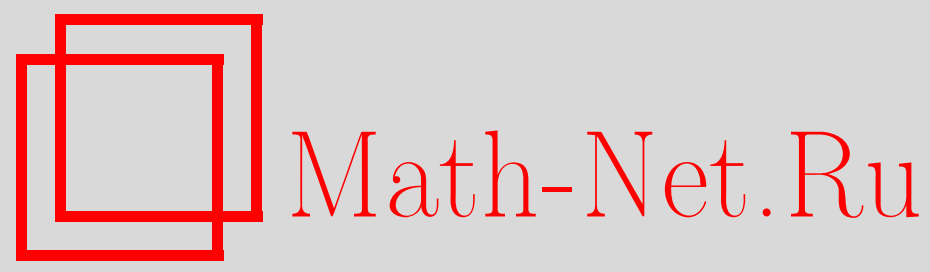

Н. В. Смирнов, Синтез идентификаторов состояния в задаче многопрограммной стабилизации билинейных систем, Матем. заметки, 2002, том 72, выпуск 4, 535-546

DOI: https://doi.org/10.4213/mzm443

Использование Общероссийского математического портала Math-Net.Ru подразумевает, что вы прочитали и согласны с пользовательским соглашением http://www.mathnet.ru/rus/agreement

Параметры загрузки:

IP : 54.164 .48 .24

26 апреля 2023 г., 15:08:28

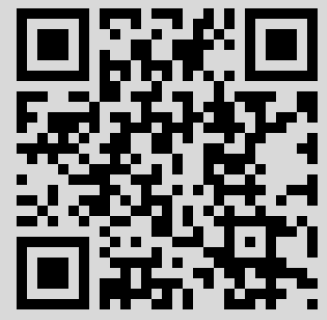




\section{СИНТЕЗ ИДЕНТИФИКАТОРОВ СОСТОЯНИЯ В ЗАДАЧЕ МНОГОПРОГРАММНОЙ СТАБИЛИЗАЦИИ БИЛИНЕЙНЫХ СИСТЕМ}

\section{Н. В. Смирнов}

В данной работе рассматривается задача стабилизации семейства программных движений билинейной нестационарной системы с применением нелинейного идентификатора состояния системы полного порядка и нелинейного идентификатора Люенбергера. Предлагается метод синтеза таких идентификаторов, а также метод построения многопрограммного стабилизирующего управления в виде нелинейной обратной связи. Доказаны теоремы о достаточных условиях существования решения поставленной задачи.

Библиография: 13 названий.

Введение. Задача стабилизации программных движений управляемых динамических систем является одной из основных в современной математической теории управления. Суть ее состоит в следующем. Пусть для некоторого управляемого объекта, движение которого описывается системой обыкновенных дифференциальных уравнений с управляющими параметрами в правых частях, построено программное управление, обеспечивающее вьполнение объектом заданного программного движения. Требуется рассчитать дополнительные управляющие воздействия, обеспечивающие этому движению асимптотическую устойчивость. Такие управляюшие воздействия принято называть стабилизирующими управлениями.

Процесс построения стабилизирующих управлений, в соответствии с теорией устойчивости, начинается с вывода системы в отклонениях [1] от заданного программного движения при заданном программном управлении. Далее весь класс задач стабилизации можно условно разделить на несколько типов. Во-первых, это задачи стабилизации линейных или нелинейных систем, в зависимости от природы исходного управляемого объекта. Во-вторых, это задачи с полной или с неполной обратной связью, в зависимости от возможностей измерительных приборов выдавать информацию о векторе фазового состояния системы в отклонениях. Основная особенность случая неполной обратной связи состоит в том, что при построении стабилизирующего управления недоступньй вектор отклонений заменяют его асимптотической оценкой. Для получения таких оценок строится так называемый асимптотический идентификатор (наблюдатель) [2], [3], представляющий собой динамическую управляемую систему, выход которой (оценка) асимптотически стремится к выходу исходной системы при совпадении управляющих воздействий. 
Следует особо отметить, что по теории асимптотических идентификаторов существует большое число публикаций от монографий до журнальных статей как теоретического, так и прикладного направления. Поэтому приведенные здесь ссылки следует рассматривать как минимально необходимые и достаточно доступные для получения вспомогательной информации.

Перейдем к обсуждению задачи многопрограммной стабилизации. В работах [4], [5] рассмотрены проблема представления правых частей систем дифференциальных уравнений, имеюших наперед заданное конечное семейство решений, а также задача синтеза управлений, которые реализуют заданную совокупность программных движений и обеспечивают их асимптотическую устойчивость по Ляпунову. В [5] особое внимание уделяется представлению таких управлений в линейных стационарных управляемых системах. Применение полученных результатов иллюстрируется в этих работах на задаче управления механическими системами, описьваемыми уравнениями Лагранжа второго рода, и на задаче управления движением заряженных частиц в электромагнитном поле.

Распространение этого подхода на классы линейных нестационарных и билинейных стационарных и нестационарных систем отражено в публикациях [6]-[8]. Следует отметить, что в этих работах речь шла о многопрограммной стабилизации в случае полной обратной связи. Актуальность определялась еще и тем, что билинейные системы являются более гибким средством аппроксимации нелинейных систем, чем линейные системы. Один из методов построения билинейных аппроксимаций представлен в [9].

Основная цель данной работы состоит в том, чтобы показать возможность синтеза идентификаторов состояния специального вида, позволяющих решать задачу многопрограммной стабилизации в классе билинейных систем в случае неполной обратной связи. Условия существования идентификатора Люенбергера, необходимого для решения этой задачи в классе линейных систем, представлены в [10].

1. Постановка задачи. Рассмотрим билинейную нестационарную управляемую систему вида

$$
\dot{\mathbf{x}}=\left(\mathbf{A}(t)+\sum_{i=1}^{r} \mathbf{B}_{i}(t) u_{i}\right) \mathbf{x}+\mathbf{F}(t),
$$

где $\mathbf{x}-n$-мерньй вектор фазового состояния; $u_{1}, \ldots, u_{r}$ - скалярные управления; $\mathbf{A}(t)$, $\mathbf{B}_{i}(t), i=1, \ldots, r,-$ вещественные, непрерывные $(n \times n)$-матрицы с ограниченньми при $t \geqslant 0$ элементами; $\mathbf{F}(t)$ - вещественная, непрерывная вектор-функция, заданная при $t \in(-\infty,+\infty)$.

Введем в рассмотрение вектор управлений $\mathbf{u}=\left(u_{1}, \ldots, u_{r}\right)^{T}$. Предположим, что для системы (1.1) построены программные управления $\mathbf{u}_{1}(t), \ldots, \mathbf{u}_{N}(t)$ в классе непрерьвных и ограниченных при $t \geqslant 0$ функций, а также соответствующие им программные движения $\mathbf{x}_{1}(t), \ldots, \mathbf{x}_{N}(t)$. Число программных движений $N$ не связано с размерностью системы (1.1) и размерностью пространства управлений.

Здесь не рассматриваются методы построения таких управлений. Для определенности будем полагать, что каждое программное управление $\mathbf{u}_{j}(t)$ и программное движение $\mathbf{x}_{j}(t)$ строятся как решение задачи по переводу системы (1.1) из заданного начального состояния в заданное конечное состояние. Таким образом, если систему (1.1) замкнуть программным управлением $\mathbf{u}_{j}(t)=\left(u_{j 1}(t), \ldots, u_{j r}(t)\right)^{T}$, то она будет иметь 
соответствующее частное решение $\mathbf{x}_{j}(t)$, отвечающее выбранным начальным и конечным данным. Другими словами, для каждой пары $\mathbf{u}_{j}(t), \mathbf{x}_{j}(t)$ имеет место следующее тождество по $t$ на интервале, где эти функции определены:

$$
\dot{\mathbf{x}}_{j}(t) \equiv \mathbf{P}_{j}(t) \mathbf{x}_{j}(t)+\mathbf{F}(t), \quad \mathbf{P}_{j}(t)=\mathbf{A}(t)+\sum_{i=1}^{r} \mathbf{B}_{i}(t) u_{j i}(t)
$$

ЗАДАчА 1. Требуется построить управление

$$
\mathbf{u}=\mathbf{u}(\mathbf{x}, t)
$$

которое реализует заданные программные движения $\mathbf{x}_{1}(t), \ldots, \mathbf{x}_{N}(t)$ и обеспечивает их асимптотическую устойчивость по Ляпунову.

Как уже отмечалось вьше, задача 1 была поставлена в [5] для линейных стационарных систем. Управление (1.3) в этой работе было построено в виде интерполяционного полинома специального вида. Для билинейной системы (1.1) задачу 1 решает

ТЕОрема 1 [8]. Пусть выполнены следующие условия:

1) программные движения $\mathbf{x}_{1}(t), \ldots, \mathbf{x}_{N}(t)$ системы (1.1) при управлениях $\mathbf{u}_{1}(t), \ldots, \mathbf{u}_{N}(t)$ различимы, т.е.

$$
\inf _{t \geqslant 0}\left\|\mathbf{x}_{i}(t)-\mathbf{x}_{j}(t)\right\|>0, \quad i \neq j
$$

2) вспомогательные линейные управляемые системы

$$
\dot{\mathbf{y}}_{j}=\mathbf{P}_{j}(t) \mathbf{y}_{j}+\mathbf{Q}_{j}(t) \mathbf{v}_{j}
$$

әде $\mathbf{Q}_{j}(t)=\left(\mathbf{B}_{1}(t) \mathbf{x}_{j}(t), \ldots, \mathbf{B}_{r}(t) \mathbf{x}_{j}(t)\right)$, стабилизируемы управлениями $\mathbf{v}_{j}=$ $\mathbf{C}_{j}(t) \mathbf{y}_{j}$, здесь $\mathbf{y}_{j}=\mathbf{x}-\mathbf{x}_{j}(t), \mathbf{v}_{j}=\mathbf{u}-\mathbf{u}_{j}(t)$ - отклонения от программных движсний и программных управлений соответственно.

Тогда существует управление (1.3), реализующее программные движения $\mathbf{x}_{1}(t)$, $\ldots, \mathbf{x}_{N}(t)$, при этом каждое из них будет асимптотически устойчиво по Ляпунову.

Доказательство этой теоремы проведено конструктивно, т.е. указан метод построения управления в следующем виде:

$$
\begin{aligned}
\mathbf{u}(\mathbf{x}, t)= & \sum_{j=1}^{N}\left(\mathbf{u}_{j}(t)+\mathbf{C}_{j}(t)\left(\mathbf{x}-\mathbf{x}_{j}(t)\right)\right. \\
& \left.-2 \mathbf{u}_{j}(t) \sum_{i=1, i \neq j}^{N} \frac{\left(\mathbf{x}_{j}(t)-\mathbf{x}_{i}(t)\right)\left(\mathbf{x}-\mathbf{x}_{j}(t)\right)}{\left(\mathbf{x}_{j}(t)-\mathbf{x}_{i}(t)\right)^{2}}\right) p_{j}(\mathbf{x}, t),
\end{aligned}
$$

где

$$
p_{j}(\mathbf{x}, t)=\prod_{i=1, i \neq j}^{N} \frac{\left(\mathbf{x}-\mathbf{x}_{i}(t)\right)^{2}}{\left(\mathbf{x}_{j}(t)-\mathbf{x}_{i}(t)\right)^{2}}
$$


В формулах $(1.4),(1.5)$ и далее выражения $\left(\mathbf{x}_{j}(t)-\mathbf{x}_{i}(t)\right)\left(\mathbf{x}-\mathbf{x}_{j}(t)\right),\left(\mathbf{x}_{j}(t)-\mathbf{x}_{i}(t)\right)^{2}$ означают скалярное произведение и скалярньй квадрат соответствующих векторов. $\mathrm{B}$ работе [8] также предложен метод построения матриц $\mathbf{C}_{j}(t)$, необходимых для стабилизации вспомогательных линейных систем из формулировки теоремы 1.

Для управления (1.4) и скалярных функций (1.5) выполнены следующие очевидные тождества:

$$
\mathbf{u}\left(\mathbf{x}_{j}(t), t\right) \equiv \mathbf{u}_{j}(t), \quad p_{j}\left(\mathbf{x}_{i}(t), t\right) \equiv 0, \quad i \neq j, \quad p_{j}\left(\mathbf{x}_{j}(t), t\right) \equiv 1
$$

В силу этих свойств система (1.1), замкнутая управлением (1.4), (1.5), имеет заданные программные движения $\mathbf{x}_{1}(t), \ldots, \mathbf{x}_{N}(t)$, т.е. будет двигаться по одному из них в случае точной установки соответствующих начальньх данных.

Таким образом, управление (1.4), (1.5) представляет собой нелинейную обратную связь, а система (1.1), замкнутая этим управлением, является нелинейным многопрограммным автоматом, который способен реализовать любое программное движение $\mathbf{x}_{j}(t)$ в зависимости от выбора начальных данных. Фактически при решении задачи 1 предполагалось, что векторы $\mathbf{x}-\mathbf{x}_{j}(t)$, являющиеся отклонениями от программных режимов, доступны для измерения. Изменим условия.

ЗАДАчА 2. Для произвольного программного движения $\mathbf{x}_{k}(t)$ из данного семейства введем вектор отклонений $\mathbf{y}_{k}(t)=\mathbf{x}-\mathbf{x}_{k}(t)$ и предположим, что задано уравнение измерительного прибора

$$
\mathbf{z}_{k}(t)=\mathbf{R y}_{k}(t)
$$

где $\mathbf{z}_{k}(t)-m$-мерньй вектор измерений; $\mathbf{R}$ - постоянная вещественная $(m \times n)$-матрица. Иногда в литературе уравнение (1.6) называют уравнением выхода [2], авектор $\mathbf{z}_{k}(t)-$ выходом системы. Зная выход $\mathbf{z}_{k}(t)$, требуется построить такую оценку $\widehat{\mathbf{y}}_{k}(t)$ вектора $\mathbf{y}_{k}(t)$, чтобы она обладала асимптотическим свойством

$$
\mathbf{y}_{k}(t)-\widehat{\mathbf{y}}_{k}(t) \rightarrow 0 \quad \text { при } t \rightarrow+\infty
$$

Свойство (1.7) по сути является требованием асимптотического покомпонентного стремления ошибки оценки отклонения от программного движения к нулевому вектору. Если такая оценка существует, то ее можно было бы использовать для синтеза управления, аналогичного управлению (1.4), (1.5). В этом и состоит задача многопрограммной стабилизации билинейной системы в случае неполной обратной связи.

Индексом $k$, в данной постановке, мы фактически выделяем одно программное движение $\mathbf{x}_{k}(t)$ из заданного набора $\mathbf{x}_{1}(t), \ldots, \mathbf{x}_{N}(t)$, т.е. то движение, которое необходимо реализовать и стабилизировать в конкретной ситуации. Вместе с тем работа системы управления должна быть универсальна по отношению к исходному семейству и зависеть лишь от начальных данных выбранного движения. Соответственно, и измерительное устройство (1.6) будет отслеживать отклонение от выбранного в данный момент программного движения $\mathbf{x}_{k}(t)$. 
2. Синтез нелинейного идентификатора полного порядка. Далее по тексту в громоздких выражениях и там, где это не мешает пониманию сути преобразований, мы не будем писать аргумент $t$ у векторных функций $\mathbf{x}_{j}, \mathbf{x}_{k}, \mathbf{y}_{k}, \ldots$

Перейдем к решению задачи 2. Замкнем систему (1.1) управлением (1.4), (1.5) и рассмотрим систему в отклонениях для программного движения $\mathbf{x}_{k}(t)$

$$
\dot{\mathbf{y}}_{k}=\left(\mathbf{P}_{k}(t)+\mathbf{Q}_{k}(t) \mathbf{C}_{k}(t)\right) \mathbf{y}_{k}+\mathbf{g}_{k}\left(\mathbf{y}_{k}\right)
$$

где

$$
\mathbf{g}_{k}\left(\mathbf{y}_{k}\right)=\left(\sum_{i=1}^{r} \mathbf{B}_{i}(t)\left(\mathbf{c}_{k i}(t) \mathbf{y}_{k}+\widetilde{u}_{k i}\right)\right) \mathbf{y}_{k}+\sum_{i=1}^{r} \mathbf{B}_{i}(t) \mathbf{x}_{k}(t) \widetilde{u}_{k i} .
$$

Вывод этой системы можно найти в [8], поэтому далее приведем лишш необходимые обозначения. Здесь $\mathbf{c}_{k i}(t) \mathbf{y}_{k}$ - скалярное произведение $i$-й строки матрицы $\mathbf{C}_{k}(t)$ и вектора отклонений $\mathbf{y}_{k}=\mathbf{x}-\mathbf{x}_{k} ; \widetilde{u}_{k i}-$ компоненты вектора

$$
\begin{aligned}
\widetilde{\mathbf{u}}_{k}= & 2\left(\mathbf{C}_{k}(t) \mathbf{y}_{k}-2 \mathbf{u}_{k}(t) s_{k}\right) s_{k}+\left(\mathbf{u}_{k}(t)+\mathbf{C}_{k}(t) \mathbf{y}_{k}-2 \mathbf{u}_{k}(t) s_{k}\right) h_{k}\left(\mathbf{y}_{k}\right) \\
& +\sum_{j=1, j \neq k}^{N}\left(\mathbf{u}_{j}(t)+\mathbf{C}_{j}(t)\left(\mathbf{y}_{k}+\mathbf{x}_{k}-\mathbf{x}_{j}\right)-2 \mathbf{u}_{j}(t) S_{j k}\right) p_{j}\left(\mathbf{y}_{k}+\mathbf{x}_{k}, t\right)
\end{aligned}
$$

где

$$
s_{k}=\sum_{i=1, i \neq k}^{N} \frac{\left(\mathbf{x}_{k}-\mathbf{x}_{i}\right) \mathbf{y}_{k}}{\left(\mathbf{x}_{k}-\mathbf{x}_{i}\right)^{2}}, \quad S_{j k}=\sum_{i=1, i \neq j}^{N} \frac{\left(\mathbf{x}_{j}-\mathbf{x}_{i}\right)\left(\mathbf{y}_{k}+\mathbf{x}_{k}-\mathbf{x}_{j}\right)}{\left(\mathbf{x}_{j}-\mathbf{x}_{i}\right)^{2}}
$$

Заметим, что $s_{k}$ и $S_{j k}$ - скалярные величины, линейно зависящие от элементов вектора $\mathbf{y}_{k}$. Кроме того, следует отметить, что при выводе системы (2.1) было использовано представление функций $p_{j}\left(\mathbf{y}_{k}+\mathbf{x}_{k}, t\right)$ и $h_{k}\left(\mathbf{y}_{k}\right)$ из [7], причем в [7] показано, что скалярная функция $h_{k}\left(\mathbf{y}_{k}\right)$ является конечной суммой однородных форм по компонентам вектора $\mathbf{y}_{k}$, начиная со второго порядка.

По построению нелинейную систему (2.1) можно рассматривать как систему, замкнутую управлением вида $\mathbf{v}_{k}=\mathbf{C}_{k}(t) \mathbf{y}_{k}$. В этом случае ее можно записать в форме

$$
\dot{\mathbf{y}}_{k}=\mathbf{P}_{k}(t) \mathbf{y}_{k}+\mathbf{Q}_{k}(t) \mathbf{v}_{k}+\mathbf{G}_{k}\left(\mathbf{y}_{k}, \mathbf{v}_{k}\right) \text {. }
$$

Идентификатор состояния для системы (2.2) предлагается строить в следующем виде:

$$
\dot{\hat{\mathbf{y}}}_{k}=\mathbf{P}_{k}(t) \widehat{\mathbf{y}}_{k}+\mathbf{Q}_{k}(t) \mathbf{v}_{k}+\mathbf{L}_{k}(t)\left(\mathbf{z}_{k}-\mathbf{R} \widehat{\mathbf{y}}_{k}\right)+\mathbf{G}_{k}\left(\widehat{\mathbf{y}}_{k}, \mathbf{v}_{k}\right)
$$

В системе (2.3) матрицы $\mathbf{P}_{k}(t), \mathbf{Q}_{k}(t), \mathbf{R}$ те же, что и в $(1.2),(1.6),(2.2)$, a $(n \times m)$-матрица $\mathbf{L}_{k}(t)$ подлежит определению. Слагаемое $\mathbf{L}_{k}(t)\left(\mathbf{z}_{k}-\mathbf{R} \widehat{\mathbf{y}}_{k}\right)$ в правой части (2.3) учитьвает качество оценки состояния. Поскольку с учетом (1.6) $\mathbf{z}_{k}-\mathbf{R} \widehat{\mathbf{y}}_{k}=\mathbf{R}\left(\mathbf{y}_{k}-\widehat{\mathbf{y}}_{k}\right)$, то в идеальной ситуации при $\widehat{\mathbf{y}}_{k} \equiv \mathbf{y}_{k}$ система (2.3) с точностью до обозначений совпадает с исходной системой $(2.2)$.

Таким образом, задача сводится к выбору матрицы $\mathbf{L}_{k}(t)$ так, чтобы имело место свойство оценки (1.7) и существовало стабилизирующее управление

$$
\mathbf{v}_{k}=\mathbf{C}_{k}(t) \widehat{\mathbf{y}}_{k}
$$


для системы (2.2).

Рассмотрим (2.2), (2.3) как одну систему. Учитывая уравнение измерителя (1.6) и вид допустимого управления (2.4), получим

$$
\left\{\begin{array}{l}
\dot{\mathbf{y}}_{k}=\mathbf{P}_{k}(t) \mathbf{y}_{k}+\mathbf{Q}_{k}(t) \mathbf{C}_{k}(t) \widehat{\mathbf{y}}_{k}+\mathbf{G}_{k}\left(\mathbf{y}_{k}, \mathbf{C}_{k}(t) \widehat{\mathbf{y}}_{k}\right), \\
\dot{\hat{\mathbf{y}}}_{k}=\mathbf{P}_{k}(t) \widehat{\mathbf{y}}_{k}+\mathbf{Q}_{k}(t) \mathbf{C}_{k}(t) \widehat{\mathbf{y}}_{k}+\mathbf{L}_{k}(t) \mathbf{R}\left(\mathbf{y}_{k}-\widehat{\mathbf{y}}_{k}\right)+\mathbf{G}_{k}\left(\widehat{\mathbf{y}}_{k}, \mathbf{C}_{k}(t) \widehat{\mathbf{y}}_{k}\right) .
\end{array}\right.
$$

В системе (2.5) сделаем неособую замену переменных

$$
\left\{\begin{array}{l}
\mathbf{y}_{k}(t)=\mathbf{y}_{k}(t) \\
\overline{\mathbf{y}}_{k}(t)=\mathbf{y}_{k}(t)-\widehat{\mathbf{y}}_{k}(t)
\end{array}\right.
$$

Переменная $\overline{\mathbf{y}}_{k}(t)$ показывает качество оценки состояния. В новых переменных система (2.5) примет вид

$$
\begin{aligned}
\left(\begin{array}{c}
\dot{\mathbf{y}}_{k} \\
\dot{\overline{\mathbf{y}}}_{k}
\end{array}\right)= & \left(\begin{array}{cc}
\mathbf{P}_{k}(t)+\mathbf{Q}_{k}(t) \mathbf{C}_{k}(t) & -\mathbf{Q}_{k}(t) \mathbf{C}_{k}(t) \\
\mathbf{O} & \mathbf{P}_{k}(t)-\mathbf{L}_{k}(t) \mathbf{R}
\end{array}\right)\left(\begin{array}{c}
\mathbf{y}_{k} \\
\overline{\mathbf{y}}_{k}
\end{array}\right) \\
& +\left(\begin{array}{c}
\mathbf{G}_{k}\left(\mathbf{y}_{k}, \mathbf{C}_{k}(t)\left(\mathbf{y}_{k}-\overline{\mathbf{y}}_{k}\right)\right) \\
\overline{\mathbf{G}}_{k}\left(\mathbf{y}_{k}-\overline{\mathbf{y}}_{k}, \mathbf{C}_{k}(t)\left(\mathbf{y}_{k}-\overline{\mathbf{y}}_{k}\right)\right)
\end{array}\right),
\end{aligned}
$$

где

$\overline{\mathbf{G}}_{k}\left(\mathbf{y}_{k}-\overline{\mathbf{y}}_{k}, \mathbf{C}_{k}(t)\left(\mathbf{y}_{k}-\overline{\mathbf{y}}_{k}\right)\right)=\mathbf{G}_{k}\left(\mathbf{y}_{k}, \mathbf{C}_{k}(t)\left(\mathbf{y}_{k}-\overline{\mathbf{y}}_{k}\right)\right)-\mathbf{G}_{k}\left(\mathbf{y}_{k}-\overline{\mathbf{y}}_{k}, \mathbf{C}_{k}(t)\left(\mathbf{y}_{k}-\overline{\mathbf{y}}_{k}\right)\right)$.

Система (2.7) состоит из двух подсистем. Первая является системой $(2.2)$, замкнутой управлением (2.4), выраженным через новые переменные (2.6), а вторая по $\overline{\mathbf{y}}_{k}$ описьвает качество оценки отклонения $\mathbf{y}_{k}$ по измерениям (1.6).

В результате задача сводится к выбору матриц $\mathbf{C}_{k}(t)$ и $\mathbf{L}_{k}(t)$ таким образом, чтобы нулевое решение системы (2.7) было асимптотически устойчиво по Ллпунову. Если такие матрицы будут найдены, то это будет означать, что, во-первых, построен асимптотический нелинейньй идентификатор вида (2.3), а, во-вторых, построено стабилизирующее управление $(2.4)$, для формирования которого используется выход $\widehat{\mathbf{y}}_{k}(t)$ идентификатора (2.3).

Перед формулировкой основного результата приведем несколько известных определений и утверждений, на которые будем ссылаться.

Рассмотрим две вспомогательные системы:

$$
\begin{aligned}
& \dot{\mathbf{x}}_{1}=\mathbf{P}_{k}(t) \mathbf{x}_{1}+\mathbf{Q}_{k}(t) \mathbf{u}_{1} \\
& \dot{\mathbf{x}}_{2}=-\mathbf{P}_{k}^{T}(t) \mathbf{x}_{2}+\mathbf{R}^{T} \mathbf{u}_{2}
\end{aligned}
$$

где матрищы $\mathbf{P}_{k}(t), \mathbf{Q}_{k}(t), \mathbf{R}$ те же, что и в (2.7), а векторы $\mathbf{x}_{1}, \mathbf{x}_{2}, \mathbf{u}_{1}, \mathbf{u}_{2}$ соответствующих размерностей имеют характер формальных обозначений. 
ЗАмЕЧАнИЕ 1. Если систему (2.9) замкнуть управлением вида $\mathbf{u}_{2}=\mathbf{L}_{k}^{T}(t) \mathbf{x}_{2}$, где матрица $\mathbf{L}_{k}(t)$ та же самая, что и в системе (2.7), то полученная система будет сопряженной [11] ко второй подсистеме линейного приближения в (2.7). Это обстоятельство необходимо учитьвать в дальнейшем.

Рассмотрим систему (2.8) как самостоятельньй объект. Следуя монографии [12], предположим, что при всех $t \geqslant 0$ ранг матрищы

$$
\widetilde{\mathbf{S}}(t)=\left(\mathbf{Q}_{k}(t), \mathrm{D} \mathbf{Q}_{k}(t), \ldots, \mathrm{D}^{n-1} \mathbf{Q}_{k}(t)\right)
$$

равен $n$ и реализуется на столбцах матрицы

$$
\mathbf{S}(t)=\left(\mathbf{q}_{k 1}(t), \mathrm{D} \mathbf{q}_{k 1}(t), \ldots, \mathrm{D}^{l_{1}-1} \mathbf{q}_{k 1}(t), \ldots, \mathbf{q}_{k s}(t), \mathrm{D} \mathbf{q}_{k s}(t), \ldots, \mathrm{D}^{l_{s}-1} \mathbf{q}_{k s}(t)\right)
$$

выбранных из совокупности столбцов матрицы $\widetilde{\mathbf{S}}(t)$. Здесь $\mathrm{D}=\mathbf{P}_{k}(t)-\mathbf{E}_{n} \frac{d}{d t}-$ оператор дифференцирования. Тогда для системы (2.8) справедлива следующая

Teорема $2[12]$. Пусть $\mathbf{P}_{k}(t) \in C_{t \in[0,+\infty)}^{2 n-2}, \mathbf{Q}_{k}(t) \in C_{t \in[0,+\infty)}^{2 n-1}$ u матрица $\mathbf{S}(t)$ является матрицей Ляпунова. Тогда систему (2.8) мохно преобразовать к обобщенному каноническому виду Фробениуса, а управление $\mathbf{u}_{1}=\mathbf{M}^{T}(t) \mathbf{x}_{1}$ выбрать таким образом, чтобы замкнутая система $\dot{\mathbf{x}}_{1}=\left(\mathbf{P}_{k}(t)+\mathbf{Q}_{k}(t) \mathbf{M}^{T}(t)\right) \mathbf{x}_{1}$ была правильной (приводимой) и имела наперед заданные характеристические показатели.

ОПРЕДЕЛЕНИЕ 1 [13]. $(n \times n)$-матрица $\mathbf{S}(t)$ называется матрицей Ляпунова, если

1) $\mathbf{S}(t) \in C_{t \in[0,+\infty)}^{1}$

2) $\mathbf{S}(t)$ и $\dot{\mathbf{S}}(t)$ ограничены при $t \in[0,+\infty)$;

$3)$ модуль определителя матрищы $\mathbf{S}(t)$ равномерно по $t \geqslant 0$ отделен от нуля.

ОПРЕДЕЛЕНИЕ 2. Линейную нестационарную управляемую систему, для которой выполнены условия теоремы 2 , будем назьвать стабилизируемой.

Перейдем к доказательству следующего утверждения.

ТЕОремА 3. Если системы (2.8), (2.9) стабилизируемы в смысле определения 2, то для системы (2.2) существует нелинейный асимптотический идентификатор (2.3) и стабилизирующее управление (2.4).

ДокАЗАТЕЛЬСтво. По определению 2 стабилизируемость систем (2.8), (2.9) означает, что для них существуют управления вида $\mathbf{u}_{1}=\mathbf{C}_{k}(t) \mathbf{x}_{1}$ и $\mathbf{u}_{2}=\mathbf{L}_{k}^{T}(t) \mathbf{x}_{2}$, при которых замкнутые системы имеют наперед заданные наборы характеристических показателей Ляпунова или спектры [11]. Будем считать, что для системы (2.8) построено управление $\mathbf{u}_{1}=\overline{\mathbf{C}}_{k}(t) \mathbf{x}_{1}$, при котором ее спектр отрицателен. Далее рассмотрим систему (2.9) и учтем замечание 1. Для нее построим управление вида $\mathbf{u}_{2}=\overline{\mathbf{L}}_{k}^{T}(t) \mathbf{x}_{2}$, которое обеспечивает замкнутой системе с матрищей $-\mathbf{P}_{k}^{T}(t)+\mathbf{R}^{T} \overline{\mathbf{L}}_{k}^{T}(t)$ правильность (приводимость) и наперед заданный положительньй спектр $\beta_{1} \geqslant \beta_{2} \geqslant \cdots \geqslant \beta_{n}>0$. Построенную таким образом матрищу $\overline{\mathbf{L}}(t)$ подставим во вторую подсистему в $(2.7)$. Ее матрица линейного приближения примет вид $\mathbf{P}_{k}(t)-\overline{\mathbf{L}}_{k}(t) \mathbf{R}$. Обозначим спектр 
этой матрицы через $\alpha_{1}, \ldots, \alpha_{n}$. Далее воспользуемся теоремой Перрона [11], в соответствии с которой для правильности линейной однородной системы необходимо и достаточно, чтобы ее полньй спектр (с учетом кратностей характеристических показателей) и полный спектр ее сопряженной системы были бы симметричны относительно нуля, т.е. должны иметь место равенства

$$
\alpha_{i}+\beta_{i}=0, \quad i=\overline{1, n} .
$$

Поскольку в нашем случае сопряженная система с матрицей $-\mathbf{P}_{k}^{T}(t)+\mathbf{R}^{T} \overline{\mathbf{L}}_{k}^{T}(t)$ является правильной и имеет положительный спектр, то в силу условия (2.10) линейная система с матрицей $\mathbf{P}_{k}(t)-\overline{\mathbf{L}}_{k}(t) \mathbf{R}$ также является правильной и имеет отрицательньй спектр $\alpha_{i}=-\beta_{i}, i=\overline{1, n}$.

Таким образом, построены матрицы $\overline{\mathbf{C}}_{k}(t)$ и $\overline{\mathbf{L}}_{k}(t)$, при которых система линейного приближения в (2.7) асимптотически устойчива. Далее нетрудно показать, что нелинейность в правой части (2.7) удовлетворяет условиям известной теоремы о стабилизации по линейному приближению [1]. Следовательно, при $\mathbf{C}_{k}(t)=\overline{\mathbf{C}}_{k}(t), \mathbf{L}_{k}=\overline{\mathbf{L}}_{k}(t)$ нулевое решение системы (2.7) асимптотически устойчиво по Ляпунову. Теорема доказана.

ЗАмЕчАниЕ 2. Теорема 3 дает алгоритм построения асимптотического нелинейного идентификатора вида (2.3) для каждого программного движения $\mathbf{x}_{k}(t)$ из заданного семейства. Это позволяет использовать ее для решения задачи 2. Управление (1.4), (1.5) в случае неполной обратной связи будет иметь вид

$$
\mathbf{u}(\widehat{\mathbf{x}}, t)=\sum_{j=1}^{N}\left(\mathbf{u}_{j}(t)+\overline{\mathbf{C}}_{j}(t)\left(\widehat{\mathbf{x}}-\mathbf{x}_{j}\right)-2 \mathbf{u}_{j}(t) \sum_{i=1, i \neq j}^{N} \frac{\left(\mathbf{x}_{j}-\mathbf{x}_{i}\right)}{\left(\mathbf{x}_{j}-\mathbf{x}_{i}\right)^{2}}\left(\widehat{\mathbf{x}}-\mathbf{x}_{j}\right)\right) p_{j}(\widehat{\mathbf{x}}, t),
$$

где

$$
p_{j}(\widehat{\mathbf{x}}, t)=\prod_{i=1, i \neq j}^{N} \frac{\left(\widehat{\mathbf{x}}-\mathbf{x}_{i}\right)^{2}}{\left(\mathbf{x}_{j}-\mathbf{x}_{i}\right)^{2}}
$$

В (2.11), (2.12) $\widehat{\mathbf{x}}=\widehat{\mathbf{y}}_{k}+\mathbf{x}_{k}$, где $\widehat{\mathbf{y}}_{k}-$ выход идентификатора (2.3).

3. Синтез нелинейного идентификатора Люенбергера. Основная цель данного пункта состоит в том, чтобы понизить порядок идентификатора (2.3) и найти условия, при вьполнении которых это возможно. В результате возникает следующая

ЗАДАчА 3. Пусть задано уравнение измерителя (1.6). Требуется выбрать $n-m$ линейных комбинаций компонент вектора $\mathbf{y}_{k}(t)$

$$
\mathbf{w}_{k}(t)=\mathbf{T}_{k} \mathbf{y}_{k}(t)
$$

где $\mathbf{w}_{k}(t)-(n-m)$-мерньй вектор, чтобы выполнялись следующие условия:

1) матрища $\mathbf{S}_{k}=\left(\begin{array}{c}\mathbf{T}_{k} \\ \mathbf{R}\end{array}\right)$ должна быть неособой;

2) для вектора $\mathbf{w}_{k}(t)$ должен существовать идентификатор, позволяющий находить оценку $\widehat{\mathbf{w}}_{k}(t)$, и при этом имеет место следующая асимптотика:

$$
\widehat{\mathbf{w}}_{k}(t)-\mathbf{w}_{k}(t) \rightarrow \mathbf{0} \text { при } t \rightarrow+\infty .
$$


Как и прежде, имеется в виду покомпонентное асимптотическое стремление вектора разности к нулевому вектору.

Если эта задача будет решена и $((n-m) \times n)$-матрица $\mathbf{T}_{k}$ будет найдена, то появится возможность построить оценку $\widehat{\mathbf{y}}_{k}(t)$ для вектора отклонений $\mathbf{y}_{k}(t)$. Действительно, с учетом (1.6), (3.1) имеем

$$
\left(\begin{array}{c}
\mathbf{w}_{k}(t) \\
\mathbf{z}_{k}(t)
\end{array}\right)=\left(\begin{array}{c}
\mathbf{T}_{k} \\
\mathbf{R}
\end{array}\right) \mathbf{y}_{k}(t)
$$

Из (3.3) получим

$$
\mathbf{y}_{k}(t)=\left(\begin{array}{c}
\mathbf{T}_{k} \\
\mathbf{R}
\end{array}\right)^{-1}\left(\begin{array}{c}
\mathbf{w}_{k}(t) \\
\mathbf{z}_{k}(t)
\end{array}\right)
$$

Это возможно, так как матрица $\mathbf{S}_{k}$ считается неособой по построению. Если предположить, что найден вектор $\widehat{\mathbf{w}}_{k}(t)$, то для вектора оценки $\widehat{\mathbf{y}}_{k}(t)$ получим выражение

$$
\widehat{\mathbf{y}}_{k}=\left(\begin{array}{c}
\mathbf{T}_{k} \\
\mathbf{R}
\end{array}\right)^{-1}\left(\begin{array}{c}
\widehat{\mathbf{w}}_{k}(t) \\
\mathbf{z}_{k}(t)
\end{array}\right)
$$

При выполнении условия (3.2), очевидно, будет иметь место следующая асимптотика оценки $\widehat{\mathbf{y}}_{k}(t)$ :

$$
\overline{\mathbf{y}}_{k}(t)=\left(\begin{array}{c}
\mathbf{T}_{k} \\
\mathbf{R}
\end{array}\right)^{-1}\left(\begin{array}{c}
\widehat{\mathbf{w}}_{k}(t)-\mathbf{w}_{k}(t) \\
\mathbf{0}
\end{array}\right) \rightarrow \mathbf{0} \text { при } t \rightarrow+\infty
$$

где переменная $\overline{\mathbf{y}}_{k}(t)=\widehat{\mathbf{y}}_{k}-\mathbf{y}_{k}$ описьвает качество оценки вектора $\mathbf{y}_{k}(t)$. Далее оценку (3.5) можно будет использовать для синтеза управления, аналогичного $(2.11),(2.12)$.

ЗАМЕчАНИЕ 3. В отличие от идентификатора полного порядка (2.3), которьй строится для всего вектора фазового состояния $\mathbf{y}_{k}$, идентификатор для вектора $\mathbf{w}_{k}$ будет иметь размерность $n-m$, так как информация (1.6) о векторе $\mathbf{y}_{k}$ считается достаточно точной и используется в (3.5) для формирования его оценки. Идентификаторы такого типа принято назьвать идентификаторами Люенбергера [2], [3]. Таким образом, задача 3 по сути является задачей синтеза нелинейного идентификатора Люенбергера, необходимого для многопрограммной стабилизации системы (1.1).

Перейдем к решению поставленной задачи. Вновь рассмотрим систему в отклонениях (2.2) и сделаем в ней замену переменных (3.3), чтобы получить уравнение, описьвающее изменение вектора $\mathbf{w}_{k}$. Введем обозначения для блоков следующих матриц:

$$
\begin{gathered}
\mathbf{S}_{k} \mathbf{P}_{k}(t) \mathbf{S}_{k}^{-1}=\left(\begin{array}{cc}
\mathbf{P}_{w w}^{(k)}(t) & \mathbf{P}_{w z}^{(k)}(t) \\
\mathbf{P}_{z w}^{(k)}(t) & \mathbf{P}_{z z}^{(k)}(t)
\end{array}\right), \quad \mathbf{S}_{k} \mathbf{Q}_{k}(t)=\left(\begin{array}{c}
\mathbf{Q}_{w}^{(k)}(t) \\
\mathbf{Q}_{z}^{(k)}(t)
\end{array}\right) \\
\mathbf{G}_{w}^{(k)}\left(\mathbf{w}_{k}, \mathbf{z}_{k}, \mathbf{v}_{k}\right)=\mathbf{T}_{k} \mathbf{G}_{k}\left(\mathbf{S}_{k}^{-1}\left(\begin{array}{c}
\mathbf{w}_{k} \\
\mathbf{z}_{k}
\end{array}\right), \mathbf{v}_{k}\right) \\
\mathbf{G}_{z}^{(k)}\left(\mathbf{w}_{k}, \mathbf{z}_{k}, \mathbf{v}_{k}\right)=\mathbf{R G}_{k}\left(\mathbf{S}_{k}^{-1}\left(\begin{array}{c}
\mathbf{w}_{k} \\
\mathbf{z}_{k}
\end{array}\right), \mathbf{v}_{k}\right)
\end{gathered}
$$


Система (2.2) в новых переменных примет вид

$$
\left\{\begin{array}{c}
\dot{\mathbf{w}}_{k}=\mathbf{P}_{w w}^{(k)}(t) \mathbf{w}_{k}+\mathbf{P}_{w z}^{(k)}(t) \mathbf{z}_{k}+\mathbf{Q}_{w}^{(k)}(t) \mathbf{v}_{k}+\mathbf{G}_{w}^{(k)}\left(\mathbf{w}_{k}, \mathbf{z}_{k}, \mathbf{v}_{k}\right) \\
\dot{\mathbf{z}}_{k}=\mathbf{P}_{z w}^{(k)}(t) \mathbf{w}_{k}+\mathbf{P}_{z z}^{(k)}(t) \mathbf{z}_{k}+\mathbf{Q}_{w}^{(k)}(t) \mathbf{v}_{k}+\mathbf{G}_{k}^{(k)}\left(\mathbf{w}_{k}, \mathbf{z}_{k}, \mathbf{v}_{k}\right)
\end{array}\right.
$$

Первое уравнение в (3.7) описьвает изменение вектора $\mathbf{w}_{k}(t)$, поэтому идентификатор для построения оценки $\widehat{\mathbf{w}}_{k}(t)$ будем искать в виде

$$
\dot{\widehat{\mathbf{w}}}_{k}=\mathbf{P}_{w w}^{(k)}(t) \widehat{\mathbf{w}}_{k}+\mathbf{P}_{w z}^{(k)}(t) \mathbf{z}_{k}+\mathbf{Q}_{w}^{(k)}(t) \mathbf{v}_{k}+\mathbf{G}_{w}^{(k)}\left(\widehat{\mathbf{w}}_{k}, \mathbf{z}_{k}, \mathbf{v}_{k}\right)
$$

Проблема выбора матрицы $\mathbf{T}_{k}$ с учетом обозначений (3.6) трансформируется в проблему выбора матриц $\mathbf{P}_{w w}^{(k)}(t), \mathbf{P}_{w z}^{(k)}(t), \mathbf{Q}_{w}^{(k)}(t)$.

В результате исходная задача сводится к следующей. Во-первых, необходимо указать алгоритм выбора матриц $\mathbf{P}_{w w}^{(k)}(t), \mathbf{P}_{w z}^{(k)}(t), \mathbf{Q}_{w}^{(k)}(t)$, чтобы для выхода идентификатора (3.8) $\widehat{\mathbf{w}}_{k}(t)$ вьполнялось условие (3.2); во-вторых, требуется построить матрицу $\mathbf{C}_{k}(t)$ таким образом, чтобы нулевое решение системы (2.2) при управлении (2.4), где $\widehat{\mathbf{y}}_{k}(t)$ определяется соотношением (3.5), было асимптотически устойчиво по Ляпунову.

Для решения этой задачи вьпишем систему (2.2), (3.8) с учетом (1.6):

$$
\left\{\begin{array}{l}
\dot{\mathbf{y}}_{k}=\mathbf{P}_{k}(t) \mathbf{y}_{k}+\mathbf{Q}_{k}(t) \mathbf{v}_{k}+\mathbf{G}_{k}\left(\mathbf{y}_{k}, \mathbf{v}_{k}\right), \\
\dot{\hat{\mathbf{w}}}_{k}=\mathbf{P}_{w w}^{(k)}(t) \widehat{\mathbf{w}}_{k}+\mathbf{P}_{w z}^{(k)}(t) \mathbf{R} \mathbf{y}_{k}+\mathbf{Q}_{w}^{(k)}(t) \mathbf{v}_{k}+\mathbf{G}_{w}^{(k)}\left(\widehat{\mathbf{w}}_{k}, \mathbf{R y}_{k}, \mathbf{v}_{k}\right) .
\end{array}\right.
$$

В (3.9) сделаем неособую замену переменных

$$
\left(\begin{array}{c}
\mathbf{y}_{k} \\
\overline{\mathbf{w}}_{k}
\end{array}\right)=\left(\begin{array}{cc}
\mathbf{E}_{n} & \mathbf{O} \\
-\mathbf{T}_{k} & \mathbf{E}_{n-m}
\end{array}\right)\left(\begin{array}{c}
\mathbf{y}_{k} \\
\widehat{\mathbf{w}}_{k}
\end{array}\right)
$$

где $\mathbf{E}_{n}, \mathbf{E}_{n-m}$ - единичные матрицы соответствующих размерностей, а новая переменная $\overline{\mathbf{w}}_{k}=\widehat{\mathbf{w}}_{k}-\mathbf{T}_{k} \mathbf{y}_{k}$ описьвает качество оценки вектора $\mathbf{w}_{k}(t)$. В результате получим

$$
\begin{aligned}
\left(\begin{array}{c}
\dot{\mathbf{y}}_{k} \\
\dot{\overline{\mathbf{w}}}_{k}
\end{array}\right)= & \left(\begin{array}{cc}
\underset{\mathbf{P}_{k}(t)}{\mathbf{O}} \\
-\mathbf{T}_{k} \mathbf{P}_{k}(t)+\mathbf{P}_{w w}^{(k)}(t) \mathbf{T}_{k}+\mathbf{P}_{w z}^{(k)}(t) \mathbf{R} & \mathbf{P}_{z z}^{(k)}(t)
\end{array}\right)\left(\begin{array}{c}
\mathbf{y}_{k} \\
\overline{\mathbf{w}}_{k}
\end{array}\right) \\
& +\left(\begin{array}{c}
\mathbf{Q}_{k}(t) \\
\mathbf{O}
\end{array}\right) \mathbf{v}_{k}+\left(\begin{array}{c}
\mathbf{G}_{k}\left(\mathbf{y}_{k}, \mathbf{v}_{k}\right) \\
\overline{\mathbf{G}}_{w}^{(k)}\left(\overline{\mathbf{w}}_{k}, \mathbf{y}_{k}, \mathbf{v}_{k}\right)
\end{array}\right)
\end{aligned}
$$

где

$$
\overline{\mathbf{G}}_{w}^{(k)}\left(\overline{\mathbf{w}}_{k}, \mathbf{y}_{k}, \mathbf{v}_{k}\right)=\mathbf{G}_{w}^{(k)}\left(\mathbf{T}_{k} \mathbf{y}_{k}+\overline{\mathbf{w}}_{k}, \mathbf{R} \mathbf{y}_{k}, \mathbf{v}_{k}\right)-\mathbf{T}_{k} \mathbf{G}_{k}\left(\mathbf{y}_{k}, \mathbf{v}_{k}\right)
$$

Рассмотрим матричное уравнение

$$
\mathbf{T}_{k} \mathbf{P}_{k}(t)=\mathbf{P}_{w w}^{(k)}(t) \mathbf{T}_{k}+\mathbf{P}_{w z}^{(k)}(t) \mathbf{R}
$$

Если бы матрица $\mathbf{T}_{k}$ была задана, то с учетом введенных обозначений (3.6) уравнение (3.12) было бы тождеством по $t$, а соответствующий блок матрицы системы (3.11) 
стал бы нулевым. В результате возникает следующая задача. Найти матрицу $\mathbf{T}_{k}$, удовлетворяющую уравнению (3.12) и такую, что $\operatorname{rang} \mathbf{S}_{k}=n$, а характеристические показатели Ляпунова линейной системы с матрицей $\mathbf{P}_{z z}^{(k)}(t)$ были бы отрищательны.

Предположим, что такая матрища $\mathbf{T}_{k}$ найдена. В этом случае идентификатор (3.8) можно считать построенньг, а вектор оценки $\widehat{\mathbf{w}}_{k}$ определенным. Следовательно, все готово для поиска стабилизирующего управления $(2.4),(3.5)$. В новых переменных $\mathbf{y}_{k}$ и $\overline{\mathbf{w}}_{k}$ оно имеет вид

$$
\mathbf{v}_{k}=\mathbf{C}_{k}(t)\left(\mathbf{y}_{k}+\overline{\mathbf{y}}_{k}\right)=\mathbf{C}_{k}(t)\left(\mathbf{y}_{k}+\mathbf{S}_{k w}^{-1} \overline{\mathbf{w}}_{k}\right)
$$

$\mathbf{S}_{k w}^{-1}$ - блок матрицы $\mathbf{S}_{k}^{-1}$, состояший из первых $n-m$ столбцов.

Замкнем систему (3.11) управлением (3.13) и учтем (3.12). Тогда

$$
\begin{aligned}
\left(\begin{array}{c}
\dot{\mathbf{y}}_{k} \\
\dot{\overline{\mathbf{w}}}_{k}
\end{array}\right)= & \left(\begin{array}{cc}
\mathbf{P}_{k}(t)+\mathbf{Q}_{k}(t) \mathbf{C}_{k}(t) & \mathbf{Q}_{k}(t) \mathbf{C}_{k}(t) \mathbf{S}_{k w}^{-1} \\
\mathbf{O} & \mathbf{P}_{z z}^{(k)}(t)
\end{array}\right)\left(\begin{array}{c}
\mathbf{y}_{k} \\
\overline{\mathbf{w}}_{k}
\end{array}\right) \\
& +\left(\begin{array}{c}
\mathbf{G}_{k}\left(\mathbf{y}_{k}, \mathbf{C}_{k}(t)\left(\mathbf{y}_{k}+\mathbf{S}_{k w}^{-1} \overline{\mathbf{w}}_{k}\right)\right) \\
\overline{\mathbf{G}}_{w}^{(k)}\left(\overline{\mathbf{w}}_{k}, \mathbf{y}_{k}, \mathbf{C}_{k}(t)\left(\mathbf{y}_{k}+\mathbf{S}_{k w}^{-1} \overline{\mathbf{w}}_{k}\right)\right)
\end{array}\right) .
\end{aligned}
$$

ТЕОРема 4. Пусть выполнены следующие условия:

1) вспомогательная линейная система (2.8) стабилизируема в смысле определения 2;

2) матричное уравнение (3.12) имеет решение относительно матрицы $\mathbf{T}_{k}$ такое, что $\operatorname{rang} \mathbf{S}_{k}=n$, а характеристические показатели Ляпунова линейной системы с матрицей $\mathbf{P}_{z z}^{(k)}(t)$ отрицательны.

Тогда для системы (2.2) существует нелинейный асимптотический идентификатор Люенбергера (3.8) и стабилизирующее управление (2.4), (3.5).

ДокАЗАТЕльство фактически приведено выше. Отметим лишь следующее. По определению 2 стабилизируемость системы (2.8) означает существование матрищы $\overline{\mathbf{C}}_{k}(t)$ такой, что при управлении $\mathbf{u}_{1}=\overline{\mathbf{C}}_{k}(t) \mathbf{x}_{1}$ ее спектр отрицателен и, следовательно, она асимптотически устойчива. При выполнении второго условия теоремы подсистема с матрицей $\mathbf{P}_{z z}^{(k)}(t)$ в линейном приближении системы (3.14) также асимптотически устойчива. При таком выборе матриц $\overline{\mathbf{C}}_{k}(t)$ и $\mathbf{P}_{z z}^{(k)}(t)$ все линейное приближение в $(3.14)$ асимптотически устойчиво. Далее нетрудно убедиться в том, что нелинейность в правой части (3.14) удовлетворяет условиям теоремы о стабилизации по линейному приближению. Теорема доказана.

ЗАМЕЧАниЕ 4. Вопрос о существовании решения уравнения (3.12) является основным, если речь идет о конкретном приложении. Следует иметь в виду, что многое в данной ситуации зависит от вида матрицы $\mathbf{P}_{k}(t)$. В монографии [2] предложен методпоиска решения аналогичного уравнения для линейных систем с постоянньми коэффициентами. Он основан, во-первых, на приведении системы к каноническому виду Фробениуса, a, во-вторых, предполагает наперед заданную асимптотику идентификатора через соответствующее определение его спектра, т.е. через задание собственных чисел матрицы $\mathbf{P}_{z z}^{(k)}$, которая будет постоянной для стационарной системы. Эта схема работает и 
в нашем случае. Проблема приведения нестационарной линейной системы к обобщенному виду Фробениуса рассмотрена в [12], а набор собственных чисел стационарного случая соответствует набору характеристических показателей Ляпунова для системы с матрицей $\mathbf{P}_{z z}^{(k)}(t)$ в данной ситуации.

ЗАмЕчАниЕ 5. Теорема 4 дает алгоритм построения асимптотического нелинейного идентификатора Люенбергера вида (3.8) для каждого программного движения $\mathbf{x}_{k}(t)$ из заданного семейства. Это позволяет использовать ее для решения задачи многопрограммной стабилизации в случае неполной обратной связи. Управление (1.4), (1.5) будет также иметь вид (2.11), (2.12). Существенное отличие однако в том, что вектор $\widehat{\mathbf{y}}_{k}$ определяется по формуле (3.5), где $\widehat{\mathbf{w}}_{k}$ - выход идентификатора (3.8).

\section{СПИСОК ЦИТИРОВАННОЙ ЛИТЕРАТУРЫ}

[1] Зубов В. И. Лекции по теории управления. М.: Наука, 1975.

[2] Андреев Ю. Н. Управление конечномерными линейными объектами. М.: Наука, 1976.

[3] Luenberger D. G. Observers for multivariable systems // IEEE Trans. Autom. Control. AC-11.. № 2. P. 190-197.

[4] Зубов В.И. Интерполяция систем дифференциальных уравнений // Докл. АН СССР. 1991. T. 318. №1. C. 28-31.

[5] Зубов В.И. Синтез многопрограммных устойчивых управлений // Докл. АН СССР. 1991. T. 318. № 2. C. $274-277$.

[6] Smirnov N.V., Smirnova T.E. The problem of the stabilization for some programmed motions of the bilinear stationary system // Proc. of Third Int. Workshop: "Beam Dynamics \& Optimization". St-Petersburg: NIICh SPbSU, 1997. P. 258-263.

[7] Смирнов Н.В., Смирнова Т. Е. Стабилизация семейства программных движений билинейной нестационарной системы // Вест. СПбГУ. Сер. 1. 1998. Т. 2. № 8. С. 70-75.

[8] Смирнов Н.В., Смирнова Т. Е. Синтез многопрограммных управлений в билинейных системах // ПММ. 2000. Т. 64. №6. С. 929-932.

[9] Svoronos S., Stephanopolos G., Aris R. Bilinear approximation of general non-linear dynamic systems with linear inputs // Int. J. Control. 1980. V. 31. № 1. P. 109-126.

[10] Smirnov N. V. Synthesis of multiprogrammed stable controls using the Luenberger observer // Prep. of 11th IFAC Int. Workshop "Control Applications of Optimization". V. 1. St-Petersburg: NIICh SPbSU, 2000. P. 317-320.

[11] Демидович Б. П. Лекции по математической теории устойчивости. М.: Наука, 1967.

[12] Смирнов Е.Я. Некоторые задачи математической теории управления. Л.: ЛГУ, 1981.

[13] Гантмахер Ф.Р. Теория матриц. М.: Наука, 1988.

Санкт-Петербургский государственный университет

Поступило

E-mail: Nikolay.Smirnov@pobox.spbu.ru

20.10 .2000

Исправленный вариант

10.11.2001 